\title{
Incidence of diabetes mellitus in women following impaired glucose tolerance in pregnancy is lower than following impaired glucose tolerance in the non-pregnant state
}

\author{
D.J. Pettitt, K. M. Venkat Narayan, R. L.Hanson, W. C. Knowler \\ Diabetes and Arthritis Epidemiology Section, Phoenix Epidemiology and Clinical Research Branch, \\ National Institute of Diabetes and Digestive and Kidney Diseases, Phoenix, Arizona, USA
}

\begin{abstract}
Summary Impaired glucose tolerance (IGT), which is asymptomatic and requires a glucose tolerance test for detection, is a well-known risk factor for diabetes mellitus. Outside the research setting it is rarely identified in people who lack specific risk factors for diabetes except during pregnancy, at which time screening with an oral glucose challenge is a routine procedure. A 75-g oral glucose tolerance test was performed during the latter part of pregnancy or during a routine epidemiology survey in 15-39-year-old Pima Indian women with no history of abnormal glucose tolerance. Those with IGT by World Health Organization criteria were included in this study. Diabetes incidence in women was compared between those whose IGT was first detected during pregnancy and those who were not pregnant when IGT was first recognized. Seventeen of 73 pregnant women and 114 of
\end{abstract}

244 non-pregnant women developed diabetes within 10 years. When controlled for plasma glucose concentration, age, body mass index, parity and duration of follow-up, those who were not pregnant were at higher risk of developing diabetes than those who were pregnant (hazard rate ratio $=1.71,95 \%$ confidence interval $=1.01-2.91)$. Previous studies had reported that women with IGT during pregnancy are at higher risk of diabetes than women with normal glucose tolerance. This study suggests that women with IGT during pregnancy are at lower risk than non-pregnant women with a similar plasma glucose concentration who, in the clinical setting, are likely to remain unrecognized. [Diabetologia (1996) 39: 1334-1337]

Keywords Diabetes mellitus incidence, impaired glucose tolerance, pregnancy.
Gestational diabetes mellitus or abnormal glucose tolerance during pregnancy has been recognized for many years as a risk factor for the future development of diabetes [1-6]. The risk of diabetes is higher in women who are older [2], are more obese [2, 4, 79], or have a more severe abnormality of glucose tolerance during the pregnancy $[2,3,6,7,9]$. Greater parity has been associated with diabetes in some [5, 8], but not all studies [9-11]. Among non-pregnant adults, impaired glucose tolerance (IGT) is also a risk factor for subsequent diabetes [12-15]. However,

Received: 15 February 1996 and in revised form: 8 May 1996

Corresponding author: Dr. D.J.Pettitt, 1550 East Indian School Road, Phoenix, AZ 85014, USA

Abbreviations: IGT, Impaired glucose tolerance; NIDDM, non-insulin-dependent diabetes mellitus. in the absence of pregnancy, glucose tolerance tests are rarely performed in young asymptomatic people as they are during pregnancy, which is a state known to affect glucose tolerance [16-18]. The comparative risks of diabetes following IGT first recognized in pregnant and non-pregnant women are therefore unknown but may be of prognostic importance. The purpose of this study is to compare the incidence of diabetes within 10 years following the recognition of IGT in women who were and in those who were not pregnant when IGT was first recognized.

\section{Subjects and methods}

Since 1965, Pima Indians living in the Gila River Indian Community in southern Arizona, a population with very high rates of non-insulin-dependent diabetes mellitus (NIDDM) [19] have participated in a longitudinal study of diabetes $[14,20]$. 
Table 1. Characteristics of women at time of recognition of IGT, duration of follow-up, parity at end of follow-up, and new cases of NIDDM

\begin{tabular}{|c|c|c|c|c|}
\hline \multirow[t]{2}{*}{ Recognition } & \multicolumn{4}{|c|}{ Pregnant at time of recognition of IGT } \\
\hline & No $(n=244)$ & & Yes $(n=73$ & \\
\hline Age (years) & $29.2 \pm 5.7$ & $15.3-39.2$ & $26.0 \pm 5.7$ & $15.7-39.9$ \\
\hline Body mass index $\left(\mathrm{kg} / \mathrm{m}^{2}\right)$ & $35.5 \pm 6.8$ & $20.9-57.2$ & $33.4 \pm 6.3$ & $23.1-50.4$ \\
\hline Number of pregnancies & $3.4 \pm 2.4$ & $1-12$ & $2.5^{\mathrm{a}} \pm 1.7$ & $1-8$ \\
\hline \multicolumn{5}{|l|}{ Follow-up } \\
\hline New cases of NIDDM & 114 & & 17 & \\
\hline
\end{tabular}

Data are mean \pm SD and range

${ }^{a}$ Includes current pregnancy

Oral glucose tolerance tests $(75 \mathrm{~g})$ are performed on women during the latter part of each pregnancy and, approximately every 2 years, on all residents of the Gila River Indian Community over the age of 5 years, regardless of whether or not they are pregnant. World Health Organization criteria [21] were used to diagnose NIDDM ( $2 \mathrm{~h}$ post-load plasma glucose level $\geq 11.1 \mathrm{mmol} / \mathrm{l})$ and IGT (2-h post-load plasma glucose $\geq 7.8 \mathrm{mmol} / \mathrm{l}$ and $<11.1 \mathrm{mmol} / \mathrm{l}$ ). The body mass index (BMI, weight divided by the square of height in $\mathrm{kg} / \mathrm{m}^{2}$ ), measured at the time of first recognition of IGT for non-pregnant women or at the first examination post pregnancy following the recognition for pregnant women, was used as a measure of obesity.

The subjects included in this study were women of at least half Pima and Tohono O'odham heritage who had IGT that was first recognized at age 15-39 years either during a pregnancy or in the non-pregnant state after at least one pregnancy, and who subsequently had at least 6 months of follow-up. Women were followed-up for up to 10 years from the first recognition of IGT - those entering the study after 1985 were followed-up for less than 10 years. The end of the follow-up period was the date of diagnosis of NIDDM or, for those not developing NIDDM within 10 years, the last non-pregnant 2yearly examination within the 10 -year period.

\section{Statistical analysis}

A proportional hazards regression model [22] was used to assess the influence of pregnancy at the time of recognition of IGT on the subsequent incidence of NIDDM. This analysis, which controls for potentially confounding variables and uses time preceding development of NIDDM as the outcome variable, accounts for the different lengths of follow-up. Parity was treated as a time-dependent variable in this model and thus increased with time in some women.

\section{Results}

Table 1 presents characteristics of the women at the time of recognition of IGT. The 244 women with IGT first identified when they were not pregnant were, on average, older, had higher plasma glucose levels, had a higher BMI, and had been pregnant more times than the 73 women whose IGT was first recognized during pregnancy ( $p<0.05$ for each). Duration of follow-up and parity at follow-up were similar in the two groups.
Table 2. Univariate predictors of NIDDM in women with IGT

\begin{tabular}{lllr}
\hline & $\begin{array}{l}\text { Hazard* } \\
\text { rate ratio }\end{array}$ & $95 \%$ CI & $p$-value \\
\hline Age (per 5 years) & 1.26 & $1.08-1.48$ & 0.004 \\
2-h plasma glucose (per mmol/l) & 1.68 & $1.41-2.01$ & $<0.001$ \\
Body mass index (per 2 kg/m²) $^{\mathrm{a}}$ & 1.05 & $1.00-1.10$ & 0.035 \\
Parity (per pregnancy) $^{\mathrm{b}}$ & 1.14 & $1.07-1.22$ & $<0.001$ \\
Not pregnant $^{\mathrm{c}}$ & 2.04 & $1.23-3.40$ & 0.006 \\
\hline
\end{tabular}

* Hazard rate ratios and $95 \%$ confidence intervals (CI) calculated from proportional hazards models. Dependent variable is NIDDM measured when not pregnant;

${ }^{a}$ measured when not pregnant

b Time-dependent variable changed during follow-up

${ }^{c}$ At recognition of IGT

Table 3. Multivariate proportional hazards model in women with IGT

\begin{tabular}{llll}
\hline & $\begin{array}{l}\text { Hazard* } \\
\text { rate ratios }\end{array}$ & 95\% CI & $p$-value \\
\hline Age (per 5 years) & 0.97 & $0.80-1.18$ & $\mathrm{NS}$ \\
2-h plasma glucose (per mmol/l) & 1.59 & $1.33-1.89$ & $<0.001$ \\
Body mass index (per 2 kg/m²) $^{\mathrm{a}}$ & 1.04 & $0.99-1.10$ & $\mathrm{NS}$ \\
Parity (per pregnancy) $^{\mathrm{b}}$ & 1.14 & $1.05-1.24$ & 0.002 \\
Not pregnant $^{\mathrm{c}}$ & 1.71 & $1.01-2.91$ & 0.048 \\
\hline
\end{tabular}

* Hazard rate ratios and $95 \%$ confidence intervals (CI) calculated from the model. Dependent variable is NIDDM measured when not pregnant;

${ }^{a}$ measured when not pregnant

$\mathrm{b}$ Time-dependent variable changed during follow-up;

${ }^{c}$ At recognition of IGT

During $0.5-10.0$ years of follow-up, NIDDM developed in $17(23.2 \%)$ women with IGT first recognized during pregnancy and in $114(46.7 \%)$ women with IGTwhile not pregnant $\left(\chi^{2}=12.7, p<0.001\right)$, but these rates may be confounded by the variables listed above.

On univariate analyses, age, plasma glucose level, BMI, parity and whether or not the woman was pregnant at time of recognition of IGT were each significantly associated with subsequent NIDDM (Table 2). On multivariate proportional hazards analysis, plasma glucose level, parity, and pregnancy at the time 


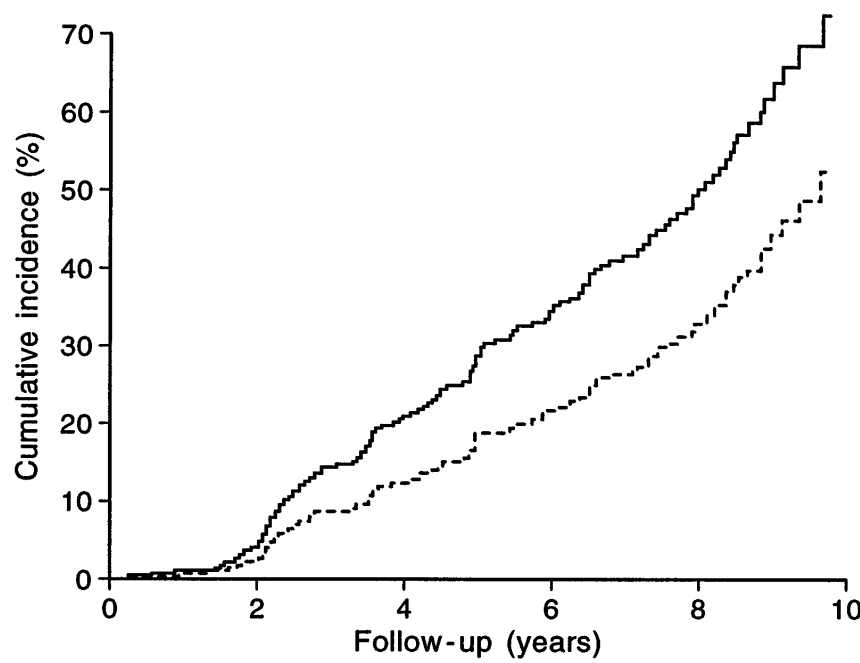

Fig. 1. Incidence of NIDDM in women who were (lower line) and those who were not (upper line) pregnant at time of the first recognition of IGT, adjusted by proportional hazards regression to the mean age (25 years), 2-h post-load glucose $(8.5 \mathrm{mmol} / \mathrm{l})$ and BMI $\left(35 \mathrm{~kg} / \mathrm{m}^{2}\right)$ at IGT recognition and the mean of the average parity during follow-up (3.5 pregnancies)

of recognition of IGT were all significantly and independently associated with subsequent NIDDM (Table 3).

Figure 1 shows the cumulative incidence of NIDDM in the women who were and were not pregnant at the time they had IGT, adjusted by proportional hazards regression to the mean age and plasma glucose level at the time, non-pregnant BMI at or following IGT and average parity during follow-up. Those who were not pregnant when IGT was first found were more likely to develop NIDDM than those who were pregnant (Table 3).

\section{Discussion}

IGT conferred a higher risk of subsequent diabetes when identified in non-pregnant women than when identified during pregnancy. No symptoms or other risk factors for diabetes, other than Pima Indian heritage, and either residence in the Gila River Indian Community or being in receipt of prenatal health care at the Community hospital, were used as criteria to select subjects for oral glucose tolerance tests. Thus, neither group was selected because of suspicion of high glucose levels. Some studies have shown that risk of diabetes increases with increasing parity [5, $8]$, but this was not found in a previous study in this population [11]. However, in the present study, the number of pregnancies used as a time-dependent variable that was incremented as subjects had pregnancies was significantly associated with the subsequent development of diabetes, and this variable was used in the multivariate proportional hazards analysis. The present analyses were limited to women who were pregnant at time of entry in the study, or who had previously had at least one pregnancy, because in this population, women who have never been pregnant are at a particularly high risk of diabetes [11]. This analysis does not exclude women with a history of menstrual irregularity, a condition also associated with higher plasma glucose levels and which increases risk of NIDDM in this population [23]. These women may have been less fertile and therefore more likely to be seen in the clinic when not pregnant than women with regular menstrual cycles.

Previous studies in this population have found abnormal glucose tolerance, in both pregnant [3] and non-pregnant [12-14] adults, to be a risk factor for subsequent NIDDM, and similar results have been found in other populations, as reviewed by Tuomilehto et al. [24]. However, unlike other studies, this study has compared the relative risk in pregnant and non-pregnant women. Systematic oral glucose tolerance tests in non-pregnant as well as pregnant women in this population have made this comparison possible. There is no reason to suspect that the findings presented in this paper would not be found in other populations, but the studies as yet have not been done.

Harris [25] has suggested that most gestational diabetes represents a pre-existing abnormality of glucose tolerance identified only because of the pregnancy, and it could be argued that this may also be the case for IGT. If so, rates of subsequent NIDDM would not be expected to differ between those who were and those who were not pregnant, and this was not the case. A likely explanation for the present findings is that women at highest risk of NIDDM developed IGT even in the absence of pregnancy while those at lower risk had normal glucose tolerance until subjected to the stress of pregnancy. This concept was expressed by Jackson [26] over 30 years ago. He stated that he did not subscribe to the idea that pregnancy was 'diabetogenic' in women with basically normal metabolism, but rather uncovered a latent or prediabetic state in these 'normal' women. This suggests that being pregnant would neither increase nor decrease the risk of NIDDM following IGT, but that the group with IGT identified during pregnancy includes women with a less severe abnormality for a given glucose, age and BMI. Since IGT is generally asymptomatic, it would remain unrecognized in the usual clinical setting where glucose tolerance tests are not routine. The predictive value of the glucose tolerance test in non-pregnant women was reported by Kjos et al. [27] who tested women in the postpartum period. Although their data are limited to women whose abnormal glucose tolerance was first identified during pregnancy, those women who also had abnormal glucose tolerance after pregnancy were at a significantly higher risk of developing diabetes within 5 years 
than were the women whose abnormality was confined to the pregnancy.

While pregnant women with abnormal glucose tolerance are more likely to develop diabetes than pregnant women with normal glucose tolerance [3], in this study they were less likely to develop diabetes than non-pregnant women with a similar degree of abnormal glucose tolerance. This may be because pregnancy is self-limited while the cause of IGT in nonpregnant women persists, or because the increased risk of subsequent diabetes associated with IGT during pregnancy is revealed by, but not caused by, the pregnancy. This may have important clinical implications as techniques in diabetes prevention are developed. Women whose IGT is identified only during pregnancy may have milder abnormalities that are less likely to progress to diabetes within 10 years, while most of the women at the greatest risk of progressing to diabetes after developing IGT are likely to remain unidentified.

Acknowledgements. We thank the members of the Gila River Indian Community for their participation in the studies, and the staff of the Diabetes and Arthritis Epidemiology Section for performing the glucose assays and for processing data.

\section{References}

1. O'Sullivan JB, Mahan CM (1964) Criteria for the oral glucose tolerance test in pregnancy. Diabetes 13: 278-285

2. O'Sullivan JB (1979) Gestational diabetes: factors influencing the rates of subsequent diabetes. In: Sutherland HW, Stowers JM (eds) Carbohydrate metabolism in pregnancy and the newborn 1978. Springer-Verlag, New York, pp 425-435

3. Pettitt DJ, Knowler WC, Baird HR, Bennett PH (1980) Gestational diabetes: infant and maternal complications of pregnancy in relation to third-trimester glucose tolerance in the Pima Indians. Diabetes Care 3: 458-464

4. Dornhorst A, Bailey PC, Anyaoku V, Elkeles RS, Johnston DG, Beard RW (1990) Abnormalities of glucose tolerance following gestational diabetes. Q J Med 77: 1219-1228

5. Henry OA, Beischer NA (1991) Long-term implications of gestational diabetes for the mother. Baillières Clinical Obstet Gynaecol 5: 461-483

6. Damm P, Kühl C, Bertelsen A, Mølsted-Pedersen L (1992) Predictive factors for the development of diabetes in women with previous gestational diabetes mellitus. Am J Obstet Gynecol 167: 607-616

7. Grant PT, Oats JN, Beischer NA (1986) The long-term follow-up of women with gestational diabetes. Aust NZ J Obstet Gynaec 26: 17-22

8. Kritz-Silverstein D, Barrett-Connor E, Wingard DL (1989) The effect of parity on the later development of non-insulin-dependent diabetes mellitus or impaired glucose tolerance. N Engl J Med 321: 1214-1219
9. Metzger BE, Cho NH, Roston SM, Radvany R (1993) Prepregnancy weight and antepartum insulin secretion predict glucose tolerance five years after gestational diabetes mellitus. Diabetes Care 16: 1598-1605

10. Boyko EJ, Alderman BW, Keane EM, Baron AE (1990) Effects of childbearing on glucose tolerance and NIDDM prevalence. Diabetes Care 13: 848-854

11. Charles MA, Pettitt DJ, McCance DR, Hanson RL, Bennett PH, Knowler WC (1994) Gravidity, obesity and noninsulin-dependent diabetes among Pima Indian women. Am J Med 97: 250-255

12. Saad MF, Knowler WC, Pettitt DJ, Nelson RG, Bennett PH (1988) Transient impaired glucose tolerance in Pima Indians: is it important? BMJ 297: 1438-1441

13. Saad MF, Knowler WC, Pettitt DJ, Nelson RG, Mott DM, Bennett PH (1988) The natural history of impaired glucose tolerance in the Pima Indians. N Engl J Med 319: 1500-1506

14. Knowler WC, Pettitt DJ, Saad MF, Bennett PH (1990) Diabetes mellitus in the Pima Indians: incidence, risk factors and pathogenesis. Diabet Metab Rev 6: 1-27

15. Stern MP, Morales PA, Valdez RA et al. (1993) Predicting diabetes: moving beyond impaired glucose tolerance. Diabetes 42: 706-714

16. Felig P (1977) Body fuel metabolism and diabetes mellitus in pregnancy. Medical Clinics of North America 61: 43-66

17. Buchanan TA, Metzger BE, Freinkel N, Bergman RN (1990) Insulin sensitivity and B-cell responsiveness to glucose during late pregnancy in lean and moderately obese women with normal glucose tolerance or mild gestational diabetes. Am J Obstet Gynecol 162: 1008-1014

18. Coustan DR (1993) Gestational diabetes. Diabetes Care 16 [Suppl 3]: 8-15

19. Knowler WC, Bennett PH, Hamman RF, Miller M (1978) Diabetes incidence and prevalence in Pima Indians: a 19fold greater incidence than in Rochester Minnesota. Am J Epidemiol 108: 497-505

20. Bennett PH, Burch TA, Miller M (1971) Diabetes mellitus in American (Pima) Indians. Lancet 2: 125-128

21. Diabetes mellitus: report of a WHO study group (1985) WHO Technical Report Series 727. World Health Organization Geneva

22. SAS Institute Inc (1989) The PHREG Procedure, Supplement to SAS/STAT User's Guide Version 6. 4th Edn. SAS Institute Inc., Cary, NC

23. Roumain JML, de Courten MP, Brodie TD, Hanson RL (1995) NIDDM is associated with menstrual irregularity in Pima Indian women. Diabetes 44 [Suppl 1]: 181A (Abstract)

24. Tuomilehto J, Knowler WC, Zimmet P (1992) Primary prevention of non-insulin-dependent diabetes mellitus. Diabet Metab Rev 8: 339-353

25. Harris MI (1988) Gestational diabetes may represent discovery of preexisting glucose intolerance. Diabetes Care 11: 402-411

26. Jackson WPU (1961) Is pregnancy diabetogenic? Lancet 2: 1369-1372

27. Kjos SL, Peters RK, Xiang A, Henry OA, Montoro M, Buchanan TA (1995) Predicting future diabetes in Latino women with gestational diabetes: utility of early postpartum glucose tolerance testing. Diabetes 44: 586-591 